\title{
THE MAX-IV DESIGN: PUSHING THE ENVELOPE
}

\author{
M. Eriksson", M. Berglund, M. Brandin, D. Kumbaro, P. Lilja, L.-J. Lindgren, L. Malmgren, M. \\ Sjöström, S. Thorin, E. Wallén, S. Werin, MAXlab, Lund, Sweden
}

Hamed Tarawneh, Sesame, Amman, Jordan

\section{Abstract}

The proposed MAX IV facility is meant as a successor to the existing MAX-lab. The accelerator part will consist of three storage rings, two new ones operated at 3 and 1.5 $\mathrm{GeV}$ respectively and the existing $700 \mathrm{MeV}$ MAX III ring. The two new rings have identical lattices and are placed on top of each other. Both these rings have a very small emittance, 0.86 and $0.4 \mathrm{~nm}$ rad respectively, and offer synchrotron radiation of very high mean brilliance. As an injector, a $3 \mathrm{GeV}$ linear accelerator is planned. The design philosophy and the special technical solutions called for are presented in this paper.

\section{INTRODUCTION}

The MAX IV facility should cover a broad spectral range of high quality spontaneous radiation. The photon spectrum should cover the region from $5 \mathrm{eV}$ to several tens of $\mathrm{keV}$. The number of straight sections for insertion devices (IDs) should be sufficiently large to cover the demand foreseen.

The demand of a broad spectral range is met by using three separate storage rings. Two new rings are to be built and they will be operated at 1.5 and $3 \mathrm{GeV}$ respectively. By having electron beams of different energies, the insertion devices can be optimised more effectively than in the one ring case. The total number of straight sections for IDs is 28 , which we believe is sufficient.

The high quality of the beam is achieved by designing the new rings to have very small emittance. The MAX III ring is operated at lower photon energies and is almost diffraction-limited in this spectral region.

The cost is optimised by placing the two new rings coaxially, on top of each other, and to use the same magnet lattices. One injector is used for all three rings. As an injector, a $3 \mathrm{GeV}$ linac is chosen. A booster is probably a more economic choice, but the linac can provide very short bunches for the generation of short pulses of spontaneous X-ray radiation and can also work as an electron source for Free Electron Lasers (FELs).

A more detailed description of the MAX IV facility can be found in ref. [1] and since the MAX III ring is described earlier [2], it will not be treated here.

\section{INJECTOR LINAC}

A S-band linac seems to be the best optimised solution for us. This linac consists of 17 equal stations, each yielding a maximum energy gain of $200 \mathrm{MeV} .15$ units should be just sufficient, but 17 will give the redundancy necessary.

Each station has one 35 MW klystron with its modulator. The modulator is preferably of the solid state kind, since a variable pulse length is needed and a high repetition rate as well. The possibility of achieving a small voltage jitter of $10^{-4}$ is currently investigated. One RF pulse compressor is used to achieve the instantaneous RF power needed.

This RF source is then feeding two RF sections, each 5 $\mathrm{m}$ long.

The electron source chosen is a pulsed thermionic gun similar to that developed at SCSS [3].

This type of gun was chosen partly because of its high performance, but most important is the reliability and robustness aspects. Topping-up injection will be used for the storage rings which imply frequent injections all around the clock. The time between injections can be used for the other purposes mentioned above. The robustness of the injector must thus have a high priority.

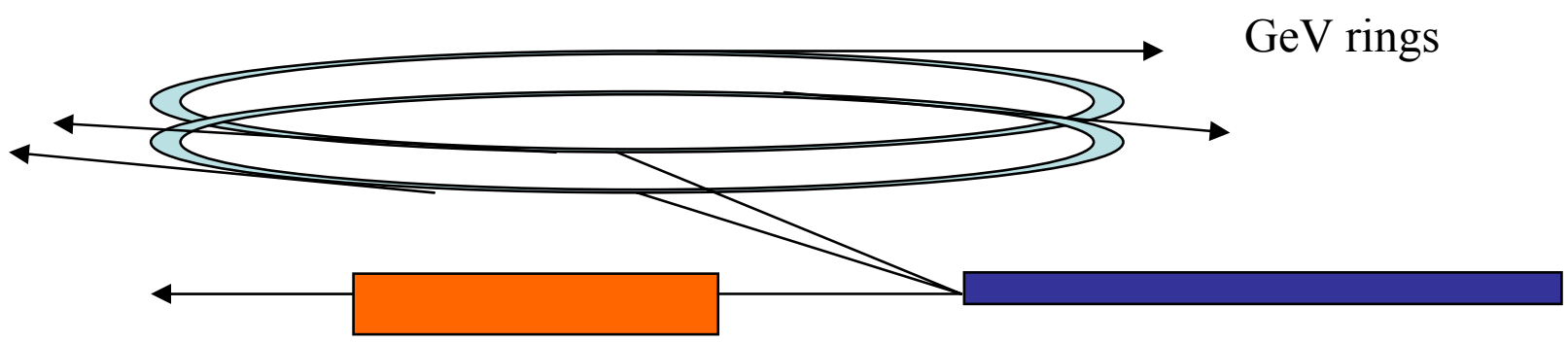

\section{Coherent radiator, SPPS}

\section{$3 \mathrm{GeV}$ linac}

Figure 1. Principle of the MAX IV facility 
Table 1: Injector Linac Parameters

\begin{tabular}{|l|l|}
\hline Max energy & $3.4 \mathrm{GeV}$ \\
\hline Total length & $250 \mathrm{~m}$ \\
\hline Energy gradient & $20 \mathrm{MeV} / \mathrm{m}$ \\
\hline Nr of RF stations & 17 \\
\hline Klystron power & $35 \mathrm{MW}$ \\
\hline RF pulse length & $1-4.5 \mu \mathrm{s}$ \\
\hline Max duty factor & $0.1 \%$ \\
\hline Max rep rate & $500 \mathrm{~s}^{-1}$ \\
\hline
\end{tabular}

\section{STORAGE RINGS}

\section{Magnet Lattice and Magnets}

To achieve a small beam emittance, a large number of dipole magnets is needed. After trying several magnet schemes, we finally ended up with a 12-fold 7 Bend Achromat lattice. The type of lattice demonstrates a high degree of stability and a large momentum acceptance for a relatively small ring. The disadvantage of having a low ratio of straight sections to total ring circumference could be neutralised by using two rings on top of each other.

This arrangement (rings on top of each other) was made possible by using the MAX III magnet technology. The magnets have a small crossection and the girders are integrated in the magnets. Several magnets (dipoles, quadrupoles) are worked out directly in solid iron blocks. The inter-magnet alignment precision becomes very high in this way.

Table 2: Ring Parameters

\begin{tabular}{|l|l|l|l|}
\hline & $\begin{array}{l}\text { MAX } \\
\text { III }\end{array}$ & $1.5 \mathrm{GeV}$ & $3 \mathrm{GeV}$ \\
\hline Nr of supercells & 8 & 12 & 12 \\
\hline Dipoles & 8 & $7 * 12$ & $7 * 12$ \\
\hline Quadrupoles & 16 & $10 * 12$ & $10^{*} 12$ \\
\hline Circumference (m) & 36 & 285 & 285 \\
\hline $\mathrm{Q}_{\mathrm{x}}$ & 3.8 & 26.6 & 26.6 \\
\hline $\mathrm{Q}_{\mathrm{y}}$ & 2.7 & 9.3 & 9.3 \\
\hline Hor emittance (nm rad) & 14 & 0.4 & 0.83 \\
\hline Energy acceptance (\%) & 3 & 4 & 4 \\
\hline Damping wigglers & 0 & 0 & 2 \\
\hline Coupling (\%) & 10 & 10 & 1 \\
\hline Energy loss / turn (keV) & 7 & 50 & 712 \\
\hline Circulating current (A) & 0.5 & 0.5 & 0.5 \\
\hline Straight section length (m) & 3 & 4.6 & 4.6 \\
\hline Nr of straight sections & 8 & 12 & 12 \\
\hline
\end{tabular}

02 Synchrotron Light Sources and FELs
Magnet lattices for small emittance rings often get crowded. To avoid this and still having a compact ring, several magnet functions are integrated into the same magnet items. So are gradients introduced into the bending magnets (this decreases the emittance as well) and the quadrupole iron poles are shaped to contain the chromaticity correcting sextupoles. The sextupoles are then located at their ideal position since the betatron functions are as most separated here and the sextupole strength needed is minimised. Octupoles are introduced in the quadrupole magnets to decrease the betatron tune variations with amplitude and these thus increase the dynamic aperture. Discrete sextupoles are so far needed to correct the vertical chromaticity, but work is underway to integrate them into the dipole ends. This will reduce the ring emittance even further.

This method of machining the pole faces of the magnets to give the desired multipole features implies a loss of flexibility. Some adjustment means are therefore introduced in the form of special windings or pole face current sheets to get the flexibility needed.

The most important ring parameters are seen in table 2 and the machine functions for one supercell in fig. 2 .

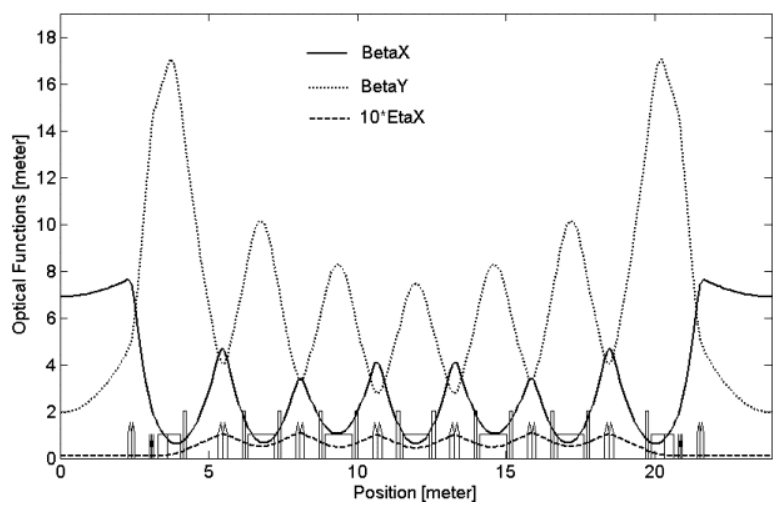

Figure 2: Machine functions for one supercell.

The parameter values for the main dipole and quadrupole magnets are seen in table 3 and 4 for the 3 $\mathrm{GeV}$ ring.

Table 3: Dipole Magnet Parameters

\begin{tabular}{|l|l|}
\hline Magnet length (m) & 0.8986 \\
\hline Magnet full gap (mm) & 24 \\
\hline Dipole field (T) & 0.907 \\
\hline Gradient (T/m) & -9.337 \\
\hline
\end{tabular}

Table 4: Quadrupole magnet parameters

\begin{tabular}{|l|l|}
\hline Magnet length $(\mathrm{m})$ & 0.3 \\
\hline Magnet bore radius $(\mathrm{mm})$ & 12 \\
\hline Gradient $(\mathrm{T} / \mathrm{m})$ & 41.2 \\
\hline Sextupole $\left(\mathrm{T} / \mathrm{m}^{2}\right)$ & 744 \\
\hline
\end{tabular}


The small magnets with the large multipole components result in small machine functions. The admittances and the energy acceptance are thus sufficiently large even if the apertures are small. The small transverse dimensions of the magnets give rather low multipole-induced magnet fields in the iron poles.

As an example, the transverse dimensions of the MAX IV dipole magnets are seen below.

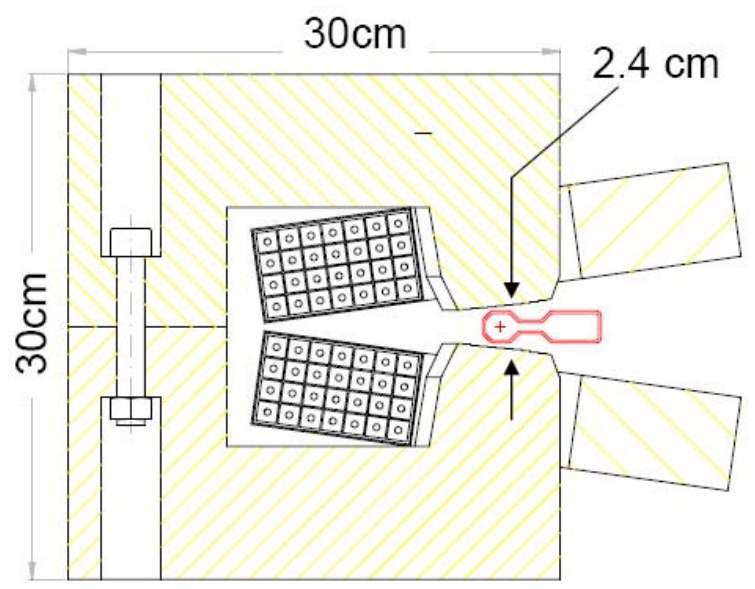

Figure 3: Half dipole magnet cross section.

\section{Aperture Needs and Dynamic Aperture}

We need a finite beam aperture for injection, gas scattering beam life-time and Touschek life-time.

The injection is favoured by using thin Lambertson septa with an effective width of $2 \mathrm{~mm}$ [4]. With a local bump injection in one long straight section and small injected beam emittance (less than $1 \mathrm{~nm} \mathrm{rad}$ ), the remaining oscillations outside the local injection bumps less than $3 \mathrm{~mm}$.

The aperture need for momentum acceptance is also small since the off-momentum functions are small. $4 \%$ energy deviation calls for less than $4 \mathrm{~mm}$ aperture.

The inelastic scattering vacuum life-time will be limited by the small gap insertion devices, not the apertures in the magnet cells.

The simulated dynamic aperture is seen below. The vacuum chamber will have an effective radius of $10 \mathrm{~mm}$, so the vacuum chamber is somewhat smaller than the dynamic aperture. The small gap insertion devices will restrict the vertical aperture to $+-2 \mathrm{~mm}$ in the $3 \mathrm{GeV}$ ring and to $+-3 \mathrm{~mm}$ in the $1.5 \mathrm{GeV}$ one.

\section{Vacuum System}

A conventional vacuum system has been worked out and described in ref. [1]. However, the successful introduction of Non-Evaporable Getter (NEG) coated vacuum chambers at several laboratories makes it very tempting to use this technology for the MAX IV storage rings in the magnet cells. A NEG-coated dipole chamber will be introduced in the MAX II ring for characterisation, hopefully this summer.

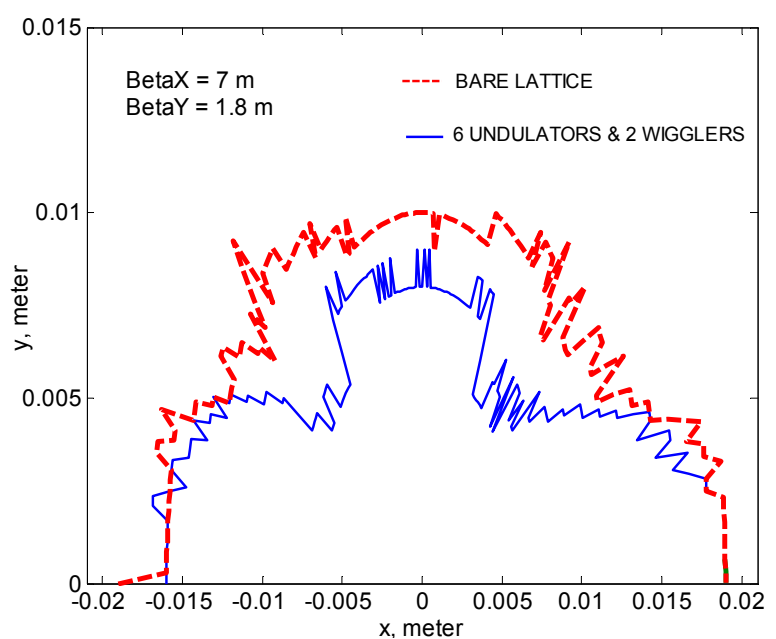

Figure 4: Dynamic aperture at the middle of the long straight sections.

\section{Radiation Properties of the Storage Rings}

The brilliance curves for a set of small gap undulators in the three rings are shown below. The brilliance of the MAX II ring, commissioned 1995, is also shown.

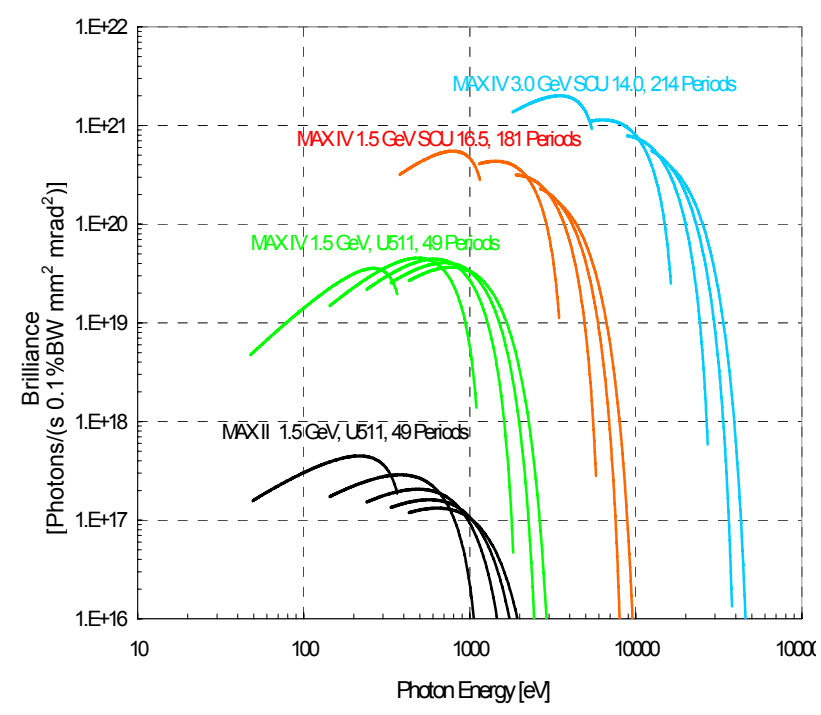

Figure 5: Brilliance plots for the MAX IV storage rings and the MAX II ring.

\section{REFERENCES}

[1] The MAX IV Conceptual Design Report http://www.maxlab.lu.se/maxlab/max4/index.html

[2] M. Eriksson, M. Bergqvist, L.-J. Lindgren, M. Brandin, M. Sjöström, S. Thorin, "Commissioning of MAX III”, EPAC'06, Edinburgh, July 2006, p. 34163417, http://www.jacow.org

[3] SCSS Conceptual Design Report http://wwwxfel.spring8.or.jp/

[4] Lars-Johan Lindgren and Bengt Anderberg, "The new septum magnets", Nucl. Instr.\& Meth A547(2005) 686-689 